\title{
The Diagnosis of Chronic Liver Disease using Machine Learning Techniques
}

\author{
Golmei Shaheamlung, Harshpreet Kaur \\ School of Computer Science and Engineering \\ Lovely Professional University Phagwara, Punjab, India
}

\begin{abstract}
In the 21st-century, the issue of liver disease has been increasing all over the world. As per the latest survey report, liver disease death toll has been rise approximately 2 million per year worldwide. The overall percentage of death by liver disease is $3.5 \%$ worldwide. Chronic Liver disease is also considered to be one of the deadly diseases, so early detection and treatment can recover the disease easily. Due to rapid advancement in Artificial intelligence (AI), like various machine learning algorithms SVM, K-mean clustering, KNN, Random forest, Logistic regression, etc., This will improve the life span of a patient suffering from Chronic Liver Disease (CLD) in early stages. The data can be obtained in a large volume due to the broad exploitation of bar codes for supreme marketable products, the mechanization of various business and government dealings, and the development in the data collection tools. This research work is based on liver disease prediction using machine learning algorithms. Liver disease prediction has various levels of steps involved, pre-processing, feature extraction, and classification. In this s research work, a hybrid classification method is proposed for liver disease prediction. And Datasets are collected from the Kaggle database of Indian liver patient records. The proposed model achieved an accuracy of $77.58 \%$. The proposed technique is implemented in Python with the Spyder tool and results are analyzed in terms of accuracy, precision, and recall.
\end{abstract}

Keywords: Artificial intelligence, Liver disease, K-means, Logistic Regression SVM, Hybrid classification

\section{Stages of Liver Disease Leading to Cirrhosis}

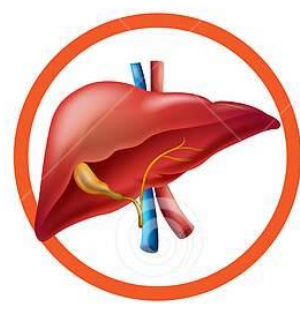

Healthy liver
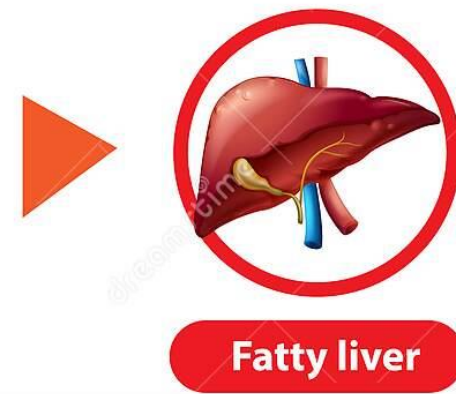

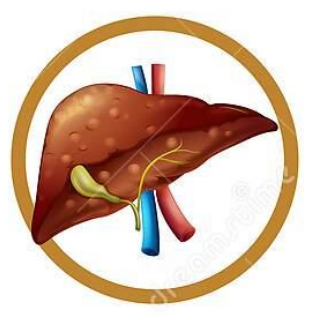

Fibrosis of liver

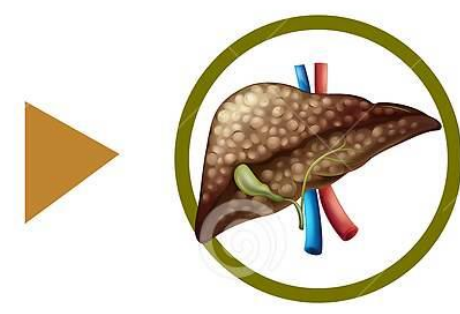

Cirrhosis of liver

Fig 1: The stages if Liver Disease 


\section{INTRODUCTION}

The capability of producing and gathering the data is growing day by day in most of the fields at present. The data can be obtained in a large volume due to the broad exploitation of bar codes for supreme marketable products, the mechanization of various business and government dealings, and the development in the data collection tools. Several databases have employed in the various applications in which government administration, scientific and engineering data management, business management comprised. Chronic liver disease starts when the scar tissue takes place of healthy tissue in the liver of the human body. Chronic liver disease is the 12th major reason for death in the United States, satges discussed in the fig 1. According to a report, chronic liver disease was the reason for $65 \%$ of liver disease increase in deaths between 1999 and 2016. The liver performs major filtering systems in our body. The main functions of the liver include removal of waste from the body, generation of amino acids, generation of blood clots, creation of new proteins, and production of bile for digestion. Moreover, it metabolizes medicines into the ingredient required for the body's healthiness. The growth of liver disorder causes muscle loss, itching, weight loss, and kidney failure in human beings. The improper functioning of the liver due to chronic liver disease causes ill effects in the entire body. These effects can be the reason for jaundice, high blood pressure, and a swollen abdomen, etc. There is the possibility of developing even more severe symptoms in case liver disease turns extreme. In such a condition, the liver can be seriously damaged and stop working, causing many toxins in the blood. Victims can even lose their life due to complications from chronic liver disease such as liver cancer. Indication of the liver is the gradual damage of liver tissue with time. There are two major categories of Liver diseases:

- Cirrhosis

- Fibrosis of the liver

As the liver gets chronically damaged, Scar tissue gradually takes place of normal operation of liver tissue. The tissue increasingly reduces the passage of blood across the liver. The damage of healthy liver tissues causes a reduction in the effective processing of nutrients, hormones, drugs, and poisons by the liver. Moreover, the liver is not able to efficiently generate proteins and other substances. The general cirrhosis signs are coughing up blood, hairlessness, vomiting, abdominal pain, back pain, weight loss, swelling, enlarge in spleen and gallbladder, nausea, pale stool, yellowing of skin and eyes color and jaundice. However, too much alcohol consumption is the key reason for this disorder. Some other causes include the use of some medicines, different types of Hepatitis, Incidents of liver failure, Viruses, fatness, and so on. Early diagnosis of liver disease is very important in order to prevent from serious damage to the liver and safe from liver transplant. Due to development in Machine learning, we can predict the liver disease in advance by applying different machine learning algorithms.

\section{Prediction of liver disease using Machine learning}

The prediction of liver diseases includes several steps. These steps are:

- Data preprocessing

- Feature selection

- Data classification

- Performance Evaluation

- Performance analysis

- Prediction 
Fig 2: General framework of chronic liver disorder prediction using Machine learning

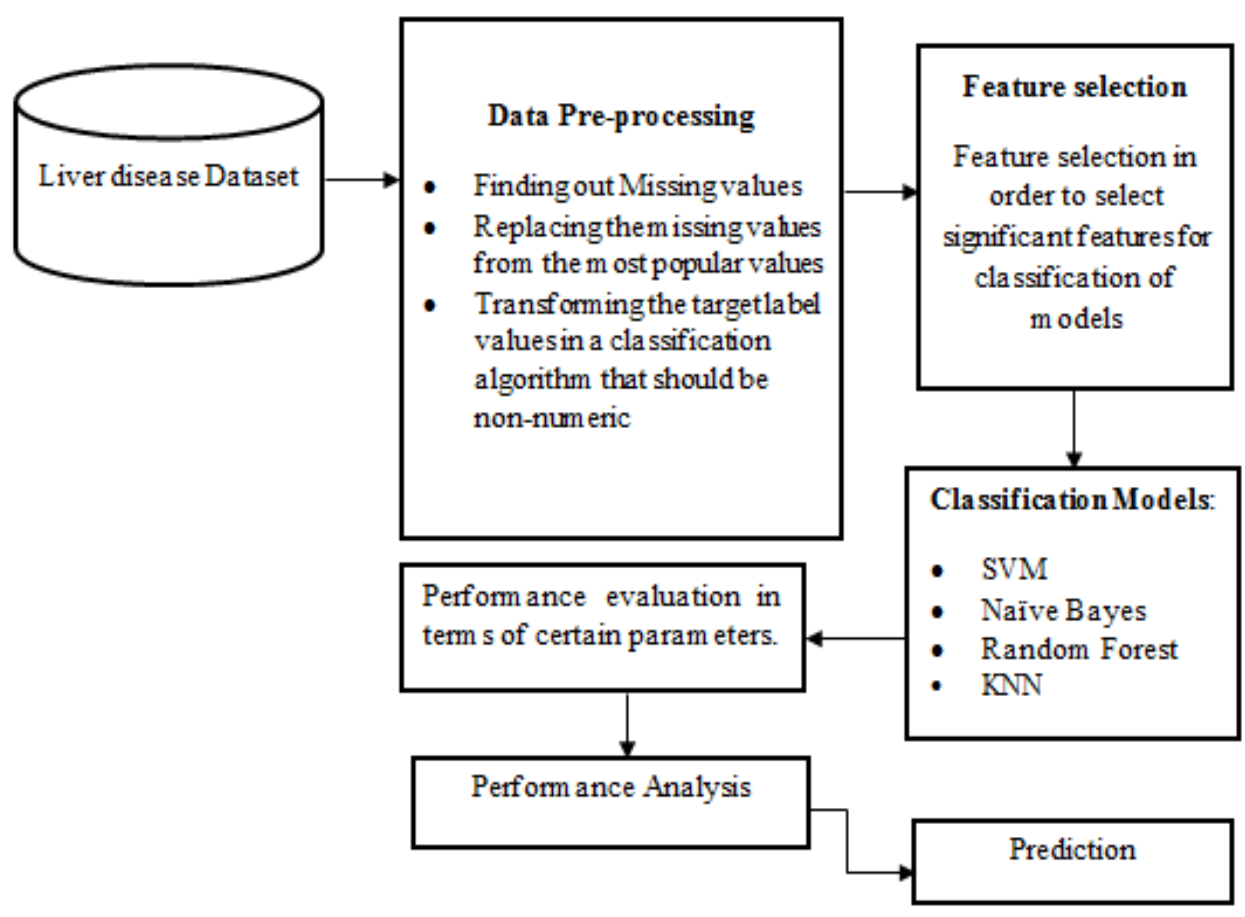

The various phases of liver disease detection are given below:-

Data pre-processing: At first, the preprocessing of data is carried out once it is collected. In this step, several tasks are performed. The collected data include many records that may have missing data or values consider age features. In general, the missing values are replaced with the nearest or closest value to their feature. And the liver disease target data are categorized into two groups i.e. group 1 represent the presence of liver disease and group 2 represent the absence of liver disease patient records [12]. The values of the target label in a classification model are converted into non-numeric. Afterward, the division of the dataset is carried out into two groups: training and testing. The data are prepared to classify the models. Once the data preprocessing is completed, the stage of selection of the models begins for classification

Feature selection: One of the main segments in chronic liver disease prediction is the selection of important features of liver disorder. In this step, several features such as age, gender hat represent the personal information of each patient is 556 Copyright $\odot$ Author selected. Some other clinical features are also collected from different medical tests.

Data classification: Classification is an important process and function in data mining. The function of the collected items assigns to the target class or category. The classification aims to get the target class to predict accurately for all case data. After data pre-processing, features are inserted in a classification model. Some popular classification models are Random Forest, SVM, and Naïve Bayes, etc.

Performance Evaluation: Different classification criteria including accuracy, precision, sensitivity, specificity, f-measure, and FRP are computed for performance evaluation of the classification.

Performance analysis: In this step, the performance of the classification model is analyzed Performance analysis: In this step, the performance of the classification model is analyzed.

Prediction: In this step, the mapping of selected features is carried out onto the training model for classifying the given features so that the liver disease can be predicted. To generate 
IT in Industry, vol. 9, no.2, 2021

predictions, a specialist doctor labels the gathered liver disease dataset. The classification is developed as a multi-class issue and the classification of medical data is carried out into different classes. Hence, every class refers to a certain category of chronic liver disorder [12]. This process can find the probabilities of a patient suffering from a liver disease based on the selected important features.

\section{LITERATURE REVIEW}

Varun Vats, et.al (2018) considered three different ML (Machine Learning) algorithms. A comparison of these algorithms had been carried out for evaluating their forecasting accuracy and computing intricacy [16]. These algorithms included AP (Affinity Propagation), K means and DBSCAN. This work was dedicated to the medical dataset based on lever disorders. This work made use of the Silhouette coefficient to measure the comparative efficiency of the considered algorithmic approaches

Vyshali J Gogi, et.al (2018) stated that the healthcare sector had a lot of data but this data was of no use [17]. This ample data required a leading analytic tool so that the hidden relationship and the valuable knowledge could be determined. The liver disease referred to the medical condition of the human liver-related to the human liver. The liver diseases led to sudden changes in health conditions that governed the functioning of the liver affecting other internal body organs. This work made use of several classification algorithms based on data mining. These algorithms included DT (Decision Tree), LD (Linear Discriminant), SVM Fine Gaussian, and LR (Logistic Regression). This work made use of Lab-based metrics of patients in the form of a liver dataset.

L. Alice Auxilia, et 1(2018) stated that the use of medical datasets had attracted the medical experts globally [18]. The use of ML (Machine Learning) algorithms was quite common as a branch of making selection expressively helpful networks for the prediction of diseases by arranging therapy-based datasets. Grouping schemes had been generally employed as a segment of the curative domain for extracting order more efficiently as compared to a signal classification model. The disorders of the Liver malady could be described as liver damage or sickness. Liver disorder can be categorized into several categories. This work made use of standard Indian liver illness patient records as a database for providing support to the researcher.

Pushpendra Kumar, et.al (2019) stated that it was a very difficult task for the doctors to detect the consequences of liver disorders on a person [19]. In general, researchers used datasets based on LFT (Liver Function Test) for implementing classification algorithms so that the predictions about liver disorders could be generated. The dataset based on ground truth had several problems such as a class imbalance in the liver disorder data. With regard to the majority classes, the classic algorithms of classification generated influenced outcomes. This work presented a new Fuzzy-ANWKNN algorithm for the successful prediction of liver disorder.

Sanjay Kumar, et.al (2018) described different classification approaches by implementing them on the dataset of patients suffering from liver diseases [20]. The main objective here was to accurately predict liver disorder by means of several data mining algorithms. This work performed the analysis using the dataset of real-time patients to build classification paradigms for the prediction of liver diseases. This work implemented five classification algorithms on the used dataset. This work analyzed different metrics such as precision, recall, and accuracy for determining the efficiency of the implemented classification models.

MafazalyaqeenHassoon, et.al (2017) devised a new approach for diagnosing liver disorders. The main aim behind devising this approach was to provide support to both medical professionals as well as patients for identifying the symptoms of malady in minimum possible time and saving valuable human life [21]. The new algorithm performed rule optimization based on the Boosted C5.0 classifier through the 
IT in Industry, vol. 9, no.2, 2021

GA(Genetic Algorithm) with the aim to reduce disease prediction time and accuracy improvement. This work made use of a genetic algorithm rather than an evolutionary approach to improve and reduce the rules of another algorithmic approach.

Nazmun Nahar and Ferdous Ara(2018), stated that their research work explores the early prediction of liver disease using various decision tree techniques. The liver disease dataset which is select for this study is consisting of attributes like total bilirubin, direct bilirubin, age, gender, total proteins, albumin, and globulin ratio[4]. The main purpose of this work is to calculate the performance of various decision tree techniques and compare their performance. The decision tree techniques used in this study are J48, LMT, Random Forest, Random tree, REPTree, Decision Stump, and Hoeffding Tree. The analysis proves that Decision Stump provides the highest accuracy than other techniques.

Thirunavukkarasu, et.al(2018), studied, the medical field produced a huge amount of healthcare data on a daily basis. The use of the ML algorithm was quite common for finding concealed information for disease detection and efficient decision-making. Significant growth in Liver diseases had been noticed with the time [27]. In several countries, these diseases were the major cause of death. This work was focused on the prediction of liver disorder with the help of several classification algorithms. These algorithms included LR, KNN, and SVM. In this work, the comparison of these algorithms had been carried in terms of accuracy rate and confusion matrix.

Nsha Arshad, et.al (2018), this work made use of data mining algorithms for training and testing the dataset for the prediction of liver disorder. This work gathered dataset from the UCI repository and developed a training dataset.

This dataset included seven different features with 345 patterns [29]. This dataset considered different sorts of blood tests that had a direct link to liver disorders. Extreme consumption of alcohol was one of the major reasons for liver disorders. The method of prediction could be
Published online 26-March-2021

Recommended on the basis of liver disorder type and got an SMO algorithm with $97.39 \%$ accuracy.

Aman Singh, et.al (2016), this work used different types of classification approaches such as LDA, DLDA, QDA, DQDA, CART, and KNN. The achieved outcomes revealed that the KNN approach based on Euclidean distance showed the best performance among all applied algorithms and generated unusually high-quality outcomes by achieving a predictive accuracy of $92.53 \%$ [30].

Sadiyah Noor Novita Alfisahrin, et.al(2013), this work was focused on identifying the patients having liver disorder on the basis of ten significant features of liver disease by means of several classification algorithms[32]. The outcomes showed that the Naïve Bayes Tree algorithm achieved maximum accuracy along with the fastest computing time. This work provided encouraging prediction results about a person being diseased or not.

Heba Ayeldeen, et.al (2015) this work made by using an ML algorithm based on DT for predicting the level of liver fibrosis in every patient [33]. The outcomes revealed that DT (Decision Tree) classifier achieved a classification accuracy of $93.7 \%$. This accuracy rate was higher than the accuracy rate reported by other investigations in the same conditions.

C. A. Prajith, et.al (2016), described the growth of scar tissue due to inflammation, infection, or injury so called liver fibrosis [35]. This disease could be the reason for liver cirrhosis. The use of various non-invasive imaging techniques was quite common for the treatment of liver fibrosis. These techniques included MRI, CT, Electrography, and ultrasound. This study was focused on the extraction of texture features from liver images of ultrasound. This work implemented various classification models such as ANN, GMM, and SVM for classifying the risk level of the liver fibrosis. SVM has a specificity of $95 \%$, the sensitivity of $93.33 \%$, and an accuracy of $94 \%$. 


\section{RESEARCH METHODOLOGY}

This work focusses on the PA (Predictive Analysis) of liver related disorders. Predictive Analysis, in short form, This research work is consists of the basic three pillars. These pillars include Pre-processing, Feature extraction, and Classification.

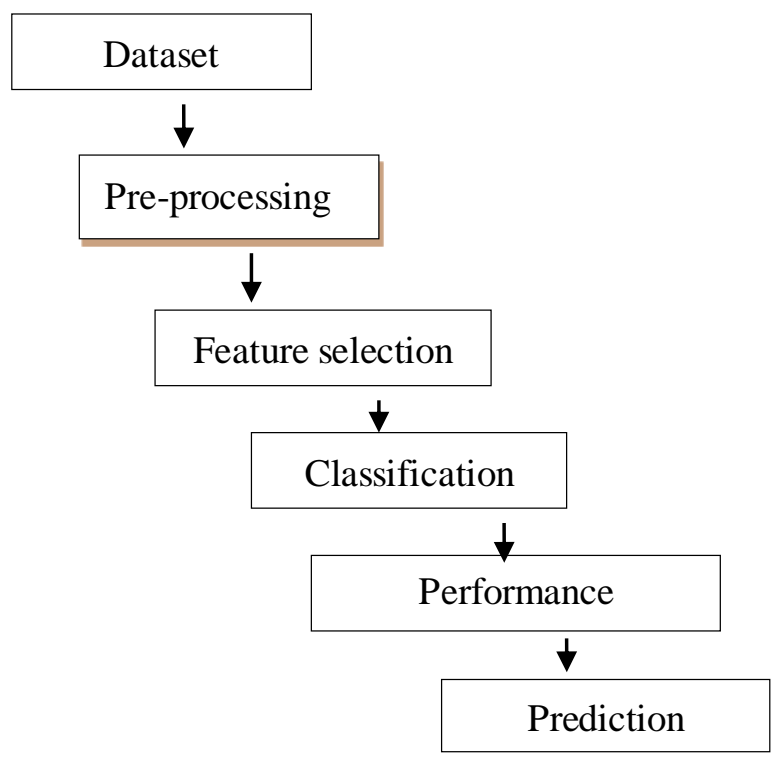

Fig 3: Process of research

1. Input Data Values: -The dataset is taken as input from the Kaggle database. The dataset has 582 instances and 10 attributes and one target (Age, Gender, TB, DB, Alkphos, Alamine Aminotransferase, Aspartate Aminotransferase, TP, ALB, A/G Ratio, and Result)

2. Data Pre-processing: In the initial stage, apply to remove missing and redundant values from the datasets.

3. Division of Input Data: -In the second step, the partitioning of whole data is carried out into two sets of training and testing. The first set of training occupies $60 \%$ part of the overall data while the rest of the part will signify the data for testing purposes.

4. Classify Data: - To predict final live or nonliver disease technique of voting classification is applied in this work.

The voting classification is the combination of logistic regression, decision tree, and $\mathrm{KNN}$ classifiers.
Logistic regression is a statistical method used for classifying dataset that includes one or more independent variables for determining the result. The classification output represents the value of one among two possible results. This approach distinguishes the software modules as defected or non-defected. This algorithm also makes use of some metrics information for classifying the software elements similar to the voting classification is the combination of logistic regression, decision tree, and $\mathrm{KNN}$ classifiers. Logistic regression is a statistical method used for classifying dataset that includes one or more independent variables are 0 more independent variables can be also interpreted using this model. $\mathrm{KNN}$ is an upfront classifier. This classifier takes into account all possible outcomes. This classifier makes use of the similarity approach for classifying a pattern. The classification of patterns is carried out on the basis of the voting majority of its neighboring patterns. A class is allotted to every pattern with the maximum number of corresponding votes and 1. This model can be used for data analysis. The association amid one dependent binary variable and one or based on a distance factor in the classification outcome. The distance between the data patterns is generally measured by Euclidean distance. The formula is given below measures distance between two neighbors $a$ and $b$, which also signifies the length of (). In Cartesian coordinates, when the coordinates of two different points in a Euclidean $\mathrm{k}$-space are specified as $\mathrm{a}=(\mathrm{a} 1, \mathrm{a} 2, \ldots$, an $)$ and $\mathrm{b}$ $=(b 1, b 2, \ldots, b n)$, then the distance $\mathrm{d}$ between points $\mathrm{a}$ and $\mathrm{b}$ is measured using the Pythagorean formula:

$$
\begin{aligned}
& d(a, b)=d(b, a) \\
& =\sqrt{\left(b_{1-} a_{1}\right)^{2}+\left(b_{2-} a_{2}\right)^{2}+\cdots+\left(b_{n}-a_{n}\right)^{2}} \\
& d(a, b)=d(b, a)=\sqrt{\sum_{i=1}^{n}\left(b_{i}-a_{i}\right)^{2}}
\end{aligned}
$$


Random Forest builds multiple decision tree and merge them together for more accuracy and stable prediction

5. Prepare training set based on all classifier

6. Apply voting classification for prediction.

7. Analyze performances in terms of Accuracy, precision, and recall

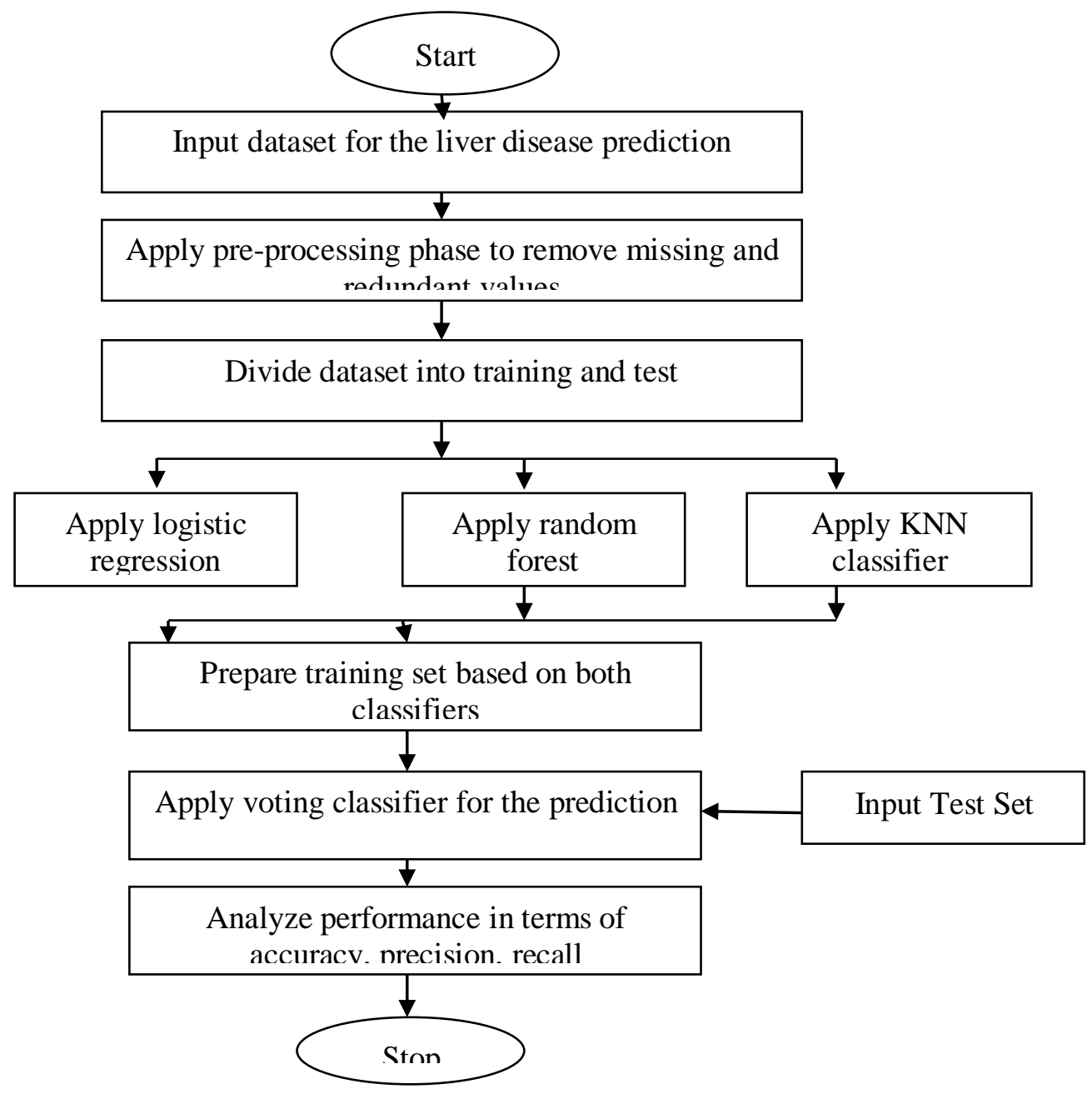

Fig. 4. Explains the flowchart of research methodology 


\section{RESULTS AND DISCUSSION}

In this research work, a hybrid model is designed for accurate prediction of liver disease. This research devises a hybrid model for making the forecast for liver disorder possible. The new prediction model combines three classifiers, Logistic Regression, Random forest, and $\mathrm{KNN}$ algorithms. The data for this purpose is taken from the Kaggle database of Indian liver patient records. This work considers different parameters in terms of accuracy, precision, and recall for analyzing the efficiency of the newly devised prediction model. This work compares the outcomes of the new prediction model against the Logistic Regression, Random forest, and KNN individually. The main aim here is to verify the outcomes generated by the new model. As per table 1 description are given, data are collected from the UCI repository. And proposed model classification performance is analyzed in terms of execution time and accuracy.

1. Accuracy: Accuracy is represented as the correctly classified number of values or points upon all total number of points multiplied by 100 . Or So, accuracy found after implementing the existing algorithm is $73.27 \%$ and in proposed work accuracy is $77.58 \%$.

$$
\text { Accuracy }=(\text { no.of } \quad T P+\text { no.of } \quad T N \quad \text { /no.of }
$$
$T P+T N+F N+T N)$

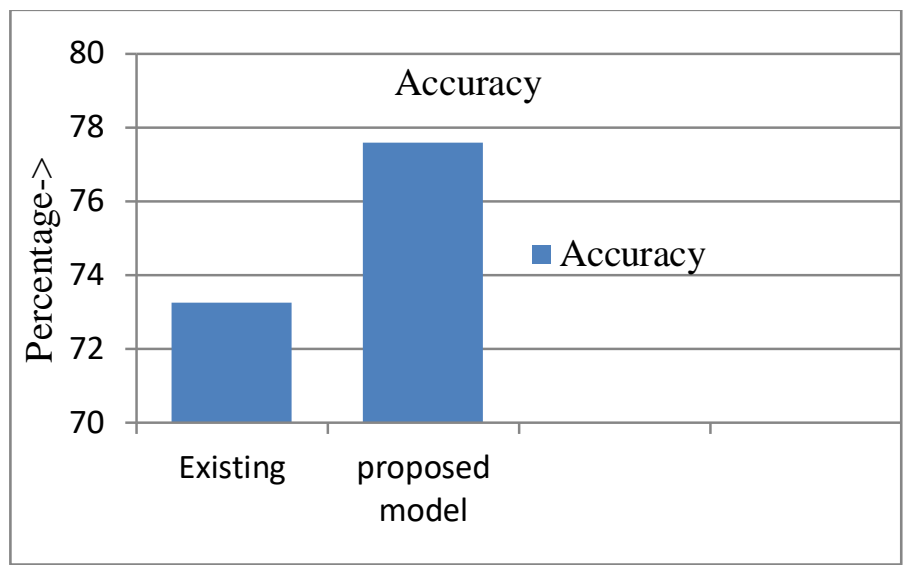

Fig. 5. Accuracy Comparison

2. Precision-Recall:-The precision-recall curve shows the tradeoff between precision and recall for different thresholds. A high area under the curve represents both high recall and high precision, where high precision relates to a low false-positive rate, and high recall relates to a low false-negative rate and good to show the interaction among them.

Precision $=($ no. of $T P /$ no.of $T P+F P)$

Recall $=($ no.of $T P /$ no. of $T P+F N)$

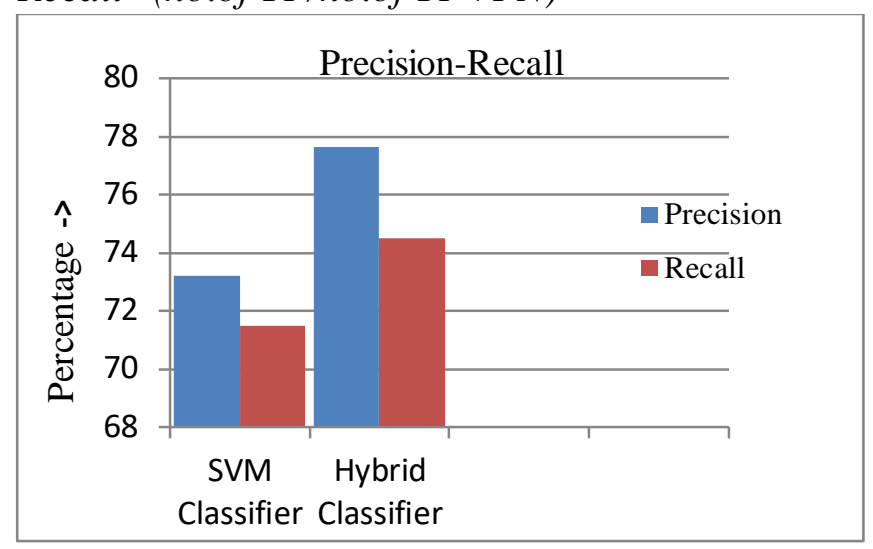

Fig. 6. Precision-Recall Comparison

3. Success Ratio: Success Ratio can be demonstrated as the ratio of number of points correctly classified to the total number of points. Eqn. 5.3defines this metric as: Success ratio $=($ Number of points correctly classified/Total number

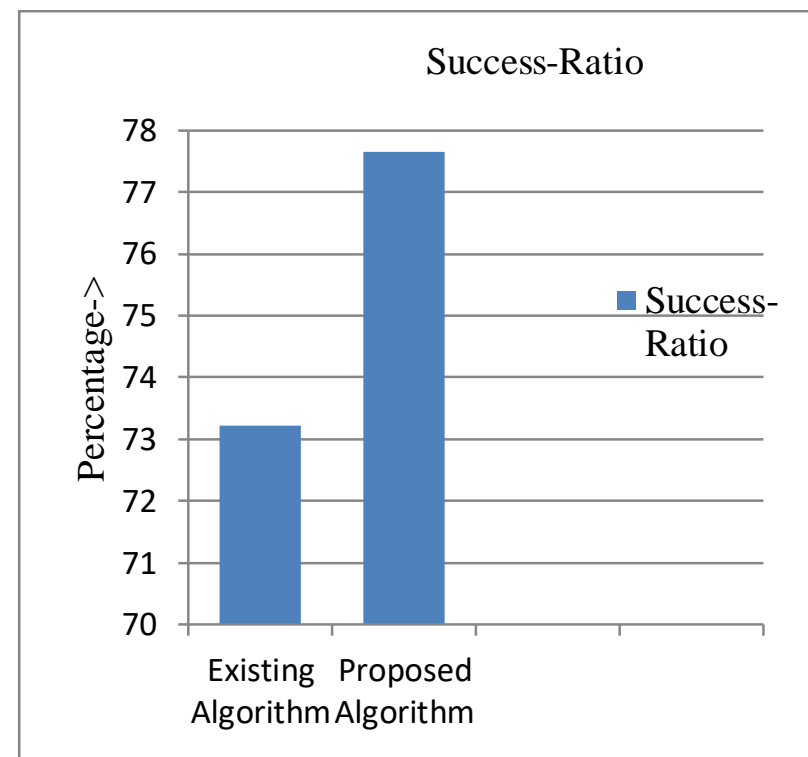

Fig. 7. Success Ratio

4. Execution Time: This metric corresponds to the difference of the end and start time of the algorithmic approach. Following Eqn. represents this metric as:

Execution time $=$ END time of the algorithmstart time of the algorithm So, execution time found after implementing existing algorithm is 
0.4 seconds and in proposed work execution time is 0.25 seconds. Table 1 shows comparison amid the existing and new algorithmic approaches with regard to certain metrics (accuracy, execution time and success ratio). In contrast to the existing algorithm, the new algorithms perform better in terms of all metric.

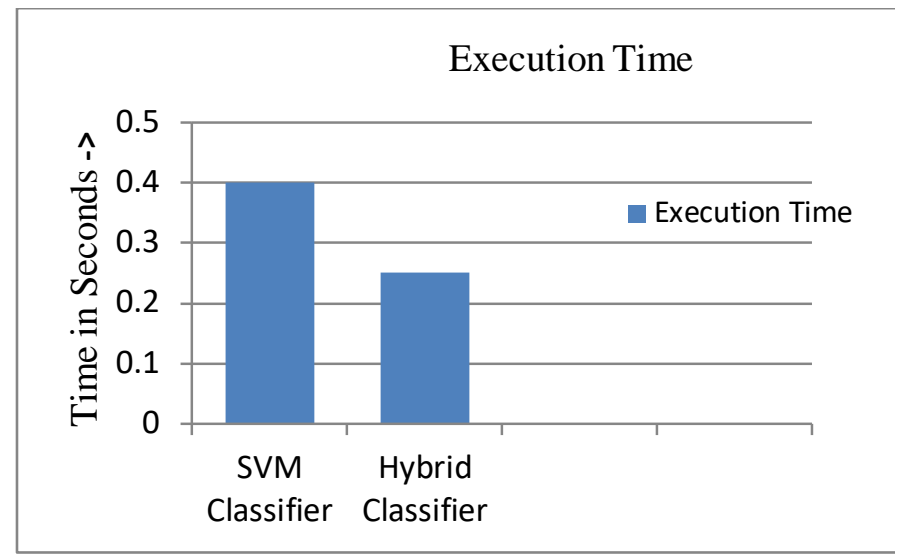

Fig. 8. Execution Time

\begin{tabular}{|l|c|c|}
\hline Parameter & $\begin{array}{c}\text { Existing } \\
\text { Algorithm }\end{array}$ & $\begin{array}{c}\text { Proposed } \\
\text { Algorithm }\end{array}$ \\
\hline Accuracy & 73.27 & 77.58 \\
\hline Precision & 73.21 & 77.65 \\
\hline Recall & 71.5 & 74.5 \\
\hline $\begin{array}{c}\text { Execution } \\
\text { time }\end{array}$ & $0.40 \mathrm{sec}$ & $0.25 \mathrm{sec}$ \\
\hline Success ratio & 0.73 & 0.77 \\
\hline
\end{tabular}

Table 1: Comparison of Existing and Proposed Algorithm

\section{CONCLUSION}

The prediction is employed to obtain the result of liver diseases with accuracy. The discovery of associations among independent and dependent variables is done with the help of prediction as suggested by its name. The hidden knowledge of liver disease is recognized and extracted using a historical liver disease database. The complex queries are responded to diagnose liver disease.
Thus, practitioners can make intelligent clinical decisions. To conclude, the approach of the predicting analysis helps in predicting future possibilities by current data. The proposed model improved by applying a combination of three classifiers, Logistic regression, Random forest, and KNN algorithm. The python is employed for the implementation of the suggested model and the result proved regarding accuracy that is achieved 77.58 percent. For the future, the execution of the clustering algorithm is performed with the hybrid classifier technique for the division of data

\section{CONFLICT OF INTEREST}

Authors have no conflict of interest with anyone. This article does not contain any studies with animals performed by any of the authors. Informed consent was obtained from all participants included in study.

\section{REFERENCES}

[1] HemlataSahu, Shalini Shrma, Seema Gondhalakar, "A Brief Overview on Data Mining Survey", International Journal of Computer Technology and Electronics Engineering (IJCTEE) Volume 1, Issue 3, 114121 (2015).

[2] Keerthi Sumiran, "An Overview of Data Mining Techniques and Their Application in Industrial Engineering", Asian Journal of Applied Science and Technology (AJAST) (Open Access Quarterly International Journal, Volume 2, Issue 2, Pages 947-953 (2018).

[3] R. Tamilselvi, S. Kalaiselvi, "An Overview of Data Mining Techniques and Applications", International Journal of Science and Research (IJSR), 2319-7064 (2013).

[4] Nazmun Nahar and Ferdous Ara, "LIVER DISEASE PREDICTION BY USING DIFFERENT DECISION TREE TECHNIQUES",International Journal of Data Mining \& Knowledge Management Process (IJDKP) Vol.8, No.2, 01-09 (2018).

[5] D. Sindhuja and R. J. Priyadarsini, "A survey on classification techniques in data mining for analyzing liver disease disorder", International Journal of Computer Science and Mobile Computing, Vol.5, no.5, 483-488 (2016). 
[6] A.S.Aneeshkumar and C.J. Venkateswaran, "Estimating the Surveillance of Liver Disorder using Classification Algorithms", International Journal of Computer Applications (0975 -8887) , Vol. 57, no. 6, 39-42 (2012).

[7] S. Dhamodharan, "Liver Disease Prediction Using Bayesian Classification", 4th National Conference on Advanced Computing, Applications \& Technologies, 1-3 (2014).

[8] G. Selvara and S. Janakiraman, "A Study of Textural Analysis Methods for the Diagnosis of Liver Disease from Abdominal Computed Tomography", International Journal of Computer Applications (0975-8887), Vol. 74, no.11, 7-13 (2013).

[9] M. Banu Priya, P. Laura Juliet, P.R. Tamilselvi, "Performance Analysis of Liver Disease Prediction Using Machine Learning Algorithms", International Research Journal of Engineering and Technology (IRJET), Volume: 05 Issue: 01, 206-211 (2018).

[10] Anju Gulia, Dr.Rajan Vohra, Praveen Rani, "Liver Patient Classification Using Intelligent Techniques", International Journal of Computer Science and Information Technologies, Vol. 5, issue 4, pp. 5110-5115 (2014).

[11] Ahmad Shaker Abdalrada, Omar Hashim Yahya, Abdul Hadi M. Alaidi, Nasser Ali Hussein, Haider TH. Alrikabi, Tahsien AlQuraishi Al-Quraishi, "A Predictive model for liver disease progression based on logistic regression algorithm", Periodicals of Engineering and Natural sciences, vol. 7, no. 3, 1255-1264 (2019).

[12] Shinya Kohara, Tomoko Tateyama, Amir Hossein Foruzan, Akira Furukawa, Shuzo Kanasak, Makoto Wakamiya, Yen-Wei Chen, "Application of statistical shape model to diagnosis of liver

disease",The 2nd International Conference on Software Engineering and Data Mining, 680683 (2010).

[13] Dong $\mathrm{Xu}$, Haiyan $\mathrm{Fu}$, Wenjuan Jiang, "Research on Liver Disease Diagnosis Based on RS_LMBP Neural Network",

12th International Conference on Computational Intelligence and Security (CIS), 646-649 (2016).

[14] P. T. Karule, S. V. Dudul, "PCA NN Based Classifier for Liver Diseases from Ultrasonic Liver Images",Second International Conference on Emerging Trends in Engineering \&
Technology, 76-80 (2009).

[15] Xin-Yu Jin, Qi-Liang Jin, Xiong Yang, “A Disease Detection Method of Liver Based on Improved Back Propagation

Neural Network", 8th International Symposium on Computational Intelligence and Design (ISCID), 111-113 (2015).

[16] Varun Vats, Lining Zhang, Sreejit Chatterjee, Sabbir Ahmed, Elvin Enziama, Kemal Tepe, “A Comparative Analysis of Unsupervised Machine Techniques for Liver Disease Prediction", IEEE International Symposium on Signal Processing and Information Technology (ISSPIT), 303-307 (2018).

[17] Vyshali J Gogi, Vijayalakshmi M.N, "Prognosis of Liver Disease: Using Machine Learning Algorithms", International Conference on Recent Innovations in Electrical, Electronics \& Communication Engineering (ICRIEECE), 875-879 (2018).

[18] L. Alice Auxilia, “Accuracy Prediction Using Machine Learning Techniques for Indian Patient Liver Disease", 2nd International Conference on Trends in Electronics and Informatics (ICOEI), 45-50 (2018).

[19] Pushpendra Kumar, Ramjeevan Singh Thakur, "Diagnosis of Liver Disorder Using Fuzzy Adaptive and Neighbor Weighted K-NN Method for LFT Imbalanced Data", International Conference on Smart Structures and Systems (ICSSS),1-5 (2019).

[20] Sanjay Kumar, Sarthak Katyal, "Effective Analysis and Diagnosis of Liver Disorder by Data Mining", International Conference on Inventive Research in Computing Applications (ICIRCA), 1047-1051 (2018).

[21] Mafazalyaqeen Hassoon, MikhakSamadiKouhi, Mariam ZomorodiMoghadam, MoloudAbdar, "Rule Optimization of Boosted C5.0 Classification Using Genetic Algorithm for Liver disease Prediction", International Conference on Computer and Applications (ICCA), 299-305 (2017).

[22] SinaBahramirad, Aida Mustapha, Maryam Eshraghi, "Classification of liver disease diagnosis: A comparative study", Second International Conference on Informatics \& Applications (ICIA), 42-46 (2013).

[23] Maria Alex Kuzhippallil, Carolyn Joseph, Kannan A, "Comparative Analysis of Machine Learning Techniques for Indian Liver Disease 
Patients", 6th International Conference on Advanced Computing and Communication Systems (ICACCS), 778-782 (2020).

[24] G. S Veena, D Sneha, Deepti Basavaraju, Tripti Tanvi, "Effective Analysis and Diagnosis of Liver Disorder", International Conference on Communication and Signal Processing (ICCSP), 0086-0090 (2018)

[25] SamyaMuhuri, Ananya Sarkar, Sambhabi Chakraborty, Susanta Chakraborty, "A Statistical Method for Prediction of Liver Disease based on the Brownian Motion Model", IEEE Region 10 Symposium (TENSYMP), 157161 (2019).

[26] Simarjot Kaur Randhawa, Ramesh Kumar Sunkaria, Anterpreet Kaur Bedi, "Prediction of Liver Cirrhosis Using Weighted Fisher Discriminant Ratio Algorithm", First International Conference on Secure Cyber Computing and Communication (ICSCCC), 184-187 (2018).

[27] k. Thirunavukkarasu, Ajay S. Singh, Md Irfan, Abhishek Chowdhury, "Prediction of Liver Disease using Classification Algorithms", 4th International Conference on Computing Communication and Automation (ICCCA), 1-3 (2018).

[28] Kanza Hamid, Amina Asif, Wajid Abbasi, DurreSabih, Fayyaz-ul-Amir Afsar Minhas, "Machine Learning with Abstention for Automated Liver Disease Diagnosis", International Conference on Frontiers of Information Technology (FIT), 356-361 (2017).

[29] Insha Arshad, Chiranjit Dutta, Tanupriya Choudhury, Abha Thakral, "Liver Disease Detection Due to Excessive Alcoholism Using Data Mining Techniques", International Conference on Advances in Computing and Communication Engineering (ICACCE), 163168 (2018).

[30] Aman Singh, Babita Pandey, "An euclidean distance based KNN computational method for assessing degree of liver damage", International Conference on Inventive Computation Technologies (ICICT), Volume: 1, 1-4 (2016).

[31] Shaker El-Sappagh, Farman Ali, Amjad Ali, AbdeltawabHendawi, Farid A. Badria, Doug Young Suh, "Clinical Decision Support System for Liver Fibrosis Prediction in Hepatitis Patients: A Case Comparison of Two Soft Computing Techniques", IEEE Access,
Volume: 6, 52911-52929 (2018).

[32] Sadiyah Noor Novita Alfisahrin, Teddy Mantoro, "Data Mining Techniques for Optimization of Liver Disease Classification", International Conference on Advanced Computer Science Applications and Technologies, 379-384 (2013).

[33] Heba Ayeldeen, Olfat Shaker, GhadaAyeldeen, Khaled M. Anwar, "Prediction of liver fibrosis stages by machine learning model: A decision tree approach", Third World Conference on Complex Systems (WCCS), 1-6 (2015).

[34] Chi Wang, Hong Song, Lei Chen, Qiang Li, Jian Yang, Xiaohua Tony $\mathrm{Hu}$, Le Zhang, "Automatic Liver Segmentation Using Multiplane Integrated Fully Convolutional Neural Networks", IEEE International Conference on Bioinformatics and Biomedicine (BIBM), 1-6 (2018).

[35] C. A. Prajith, A. Sukesh Kumar, Harish Kareem, "Supervised classification and prediction of fibrosis seriousness using ultrasonic images", IEEE International, 1-4 (2016).

[36] GolmeiShaheamlung, Harshpreet Kaur, Jimmy Singla, "A Comprehensive Review of Medical Expert Systems for Diagnosis of Chronic Liver Diseases", International Conference on Computational Intelligence and Knowledge Economy (ICCIKE), 731-735 (2019).

[37] Somaya Hashem, Gamal Esmat, Wafaa Elakel, ShahiraHabashy, Safaa Abdel Raouf, Mohamed Elhefnawi, Mohamed I. Eladawy, Mahmoud ElHefnawi, "Comparison of Machine Learning Approaches for Prediction of Advanced Liver Fibrosis in Chronic Hepatitis C Patients", IEEE/ACM Transactions on Computational Biology and Bioinformatics, Volume: 15, Issue: 3, 861-868 (2018).

[38] Anu Sebastian, Surekha Mariam Varghese, "Fuzzy logic for Child-Pugh classification of patients with cirrhosis of liver", International Conference on Information Science (ICIS), 168171 (2016).

[39] AkifumiMiyazakiy, Miho Ohsaki, Eri Taniguchi, Shigeru Katagiri ;Hideto Yokoi ; Katsuhiko Takabayashi, "Feature extraction for the prediction of liver fibrosis stages in chronic hepatitis C", TENCON IEEE Region 10 Conference, 1-5 (2012).

[40] Mahmoud Nasr, Khaled El-Bahnasy, M. 
Hamdy, Sanaa M. Kamal, "A novel model based on non-invasive methods for prediction of liver fibrosis", 13th International Computer Engineering Conference (ICENCO), 276-281 (2017).

[41] Ahmed M. Hashem, M. Emad M. Rasmy, Khaled M. Wahba, Olfat G. Shaker, "Prediction AUTHORS PROFILE

Golmei Shaheamlung, M.tech

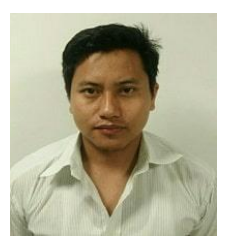

(Computer Science and Engineering), Lovely Professional University, Punjab.

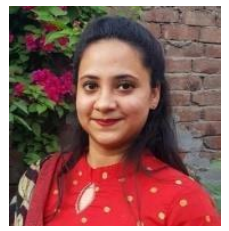

Harshpreet Kaur, Assistant Professor (Computer Science and Engineering), Lovely Professional University, Punjab of the degree of liver fibrosis using different pattern recognition techniques", 5th Cairo International Biomedical Engineering Conference, 210-214(2010). 